\title{
Evaluación del impacto de las intervenciones realizadas para mejorar el registro de valoración de enfermería al ingreso
}

\author{
M.A. Fernández Molina a , J. Gómez Robles ${ }^{b}$, E. Gabaldón Bravo ${ }^{b}$, M. Domingo Pozob, \\ M. TORRES Figueiras ${ }^{b}$ y C. TeRrés RUIZ ${ }^{b}$ \\ aDUE. Director de Enfermería. Hospital General Universitari d'Alacant. Alicant \\ ${ }^{b}$ DUE asistencial. Hospital General Universitari d'Alacant. Alicant. España.
}

\section{Resumen}

Introducción. Este artículo tiene como objetivo comparar el nivel de cumplimentación de la hoja de valoración del paciente al ingreso por el profesional de enfermería en el Hospital General Universitario de Alicante (HGUA), antes y después de las intervenciones contempladas dentro del proyecto FIS 00/1049.

Material y métodos. Estudio cuasiexperimental antes-después. Sujetos a estudio: hoja de valoración de enfermería del paciente al ingreso.

El estudio consta de 3 fases:

1. Audit de la hoja de valoración antes de las intervenciones: estudio descriptivo transversal. Muestra de 578 hojas de valoración.

2. Fase de investigación cualitativa (técnica grupo nominal y grupos de discusión) para la planificación de intervenciones.

3. Intervenciones: diseño de una nueva hoja y su guía de cumplimentación. Implantación en todo el hospital.

4. Audit tras las intervenciones: estudio descriptivo transversal. Muestra aleatorizada de 104 hojas de valoración.

Resultados. Análisis comparativo de frecuencias. Aumento general en todos los ítems estudiados, especialmente en las necesidades básicas que rondan el $90 \%$ de cumplimentación, que es el
Correspondencia: Miguel Ángel Fernández Molina. Hospital General Universitari d'Alacant.

Pintor Baeza, s/n. 03010 Alacant. España.

Correo electrónico: fernandez_mig@gva.es

Aceptado para su publicación el 16-10-2002. estipulado como óptimo. Introducción en la hoja de valoración de nuevas variables y escalas de medida.

Discusi ón. El aumento en el nivel de cumplimentación ha sido posible gracias al trabajo conjunto con los profesionales de enfermería del hospital y a la adecuación del tipo de registro (semicerrado) al momento profesional de los/as enfermeros/as del HGUA.

Palabras clave: Valoración enfermera. Documentación clínica. Registros de enfermería.

\section{I mpact of interventions to improve the admission assessment sheet}

Introduction. To compare the degree of completion of patients' admission assessment sheets by the nursing staff in the General University of Alicante Hospital (GUAH), before and after intervention strategies within the FIS project 00/1049.

Material and methods. A before-and-after quasi-experimental study was performed of patients' assessment sheets completed by nurses at admission.

There were three phases:

1. Audit of the assessment sheet before the interventions. Cross-sectional descriptive study. Sample: 578 assessment sheets.

2. Qualitative research phase (nominal group technique and discussion groups) to plan intervention strategies.

3. Interventions: design of a new sheet/form together with a guide for its correct completion. Implementation throughout the hospital.

4. Audit after interventions: cross-sectional descriptive study. Random sample of 104 assessment sheets. 
Results. Comparative analysis of frequencies. There was a general increase in all the items studied. Completion was $90 \%$ for items regarding basic needs, a result considered as optimal. New variables and measurement scales were introduced in the assessment sheet.

Discussion. Improved completion was achieved by collaboration with the hospital's nursing staff, and by adaptation of the type of record (semi-closed) to the current professional circumstances of nurses in the GUAH.

Key words: Nursing assessment. Clinical documentation. Nursing records.

\section{Introducción}

Debido a motivos culturales de la profesión enfermera, tradicionalmente, las enfermeras no dieron importancia relevante al registro de las actividades que realizaban ${ }^{1,2,3}$. Fue a partir de los años ochenta cuando comienza a aparecer un creciente interés por la documentación y los registros de enfermería, todo esto de forma paralela a la creación del título de Diplomado Universitario en Enfermería. Como repercusión de este hecho se produce un cambio en la concepción del profesional, que con anterioridad y durante un largo período histórico fue definido como un profesional subordinado a otros, con actividades delegadas, y pasó a ser un profesional con un ámbito de autonomía propio. Surge en estos momentos la necesidad de reflejar todas aquellas actividades de enfermería en documentos escritos, en los que se reflejará la autonomía y la aportación concreta de los profesionales 4 .

Este cambio conceptual no se produce por igual en todos los profesionales de enfermería del ámbito asistencial, ya que el cambio en la titulación no conllevó una formación específica en las áreas de documentación y registros de enfermería, por lo que los profesionales en la asistencia tuvieron que ser autodidactas. Por ello, a lo largo de los años esta situación provocó un déficit en la cumplimentación de los registros. Esta deficiencia, aunque por otros motivos, también es extrapolable al ámbito internacional $5,6,7,8$.

A la vista de todo ello, nuestro grupo de trabajo encaminó sus investigaciones hacia este campo.

Nuestra realidad en el momento de partida era que:

1. EI nivel de cumplimentación de la hoja de valoración del paciente al ingreso por los enfermeros era inferior al porcentaje estipulado como óptimo: $90 \%$.

2. No existía unanimidad en los datos a reflejar en este documento.
Para poder mejorar estos resultados, se presentó un trabajo como proyecto de investigación al Fondo de Investigación Sanitaria, aprobado por este organismo en el período 1999-2000.

La hipótesis de este trabajo es la siguiente: si determinamos las causas del bajo nivel de cumplimentación de la hoja de valoración de enfermería del paciente al ingreso y se actúa sobre las que son modificables, se debe obtener una mejora en los niveles de cumplimentación de la hoja.

Definimos como objeti vo: comparar el nivel de cumplimentación de la hoja de valoración del paciente al ingreso por parte de enfermeras en el Hospital General Universitario de Alicante (HGUA), antes y después de las intervenciones contempladas dentro de este proyecto.

\section{Material y métodos}

E studio cuasiexperimental antes-después de la hoja de valoración de enfermería al ingreso del paciente en el hospital.

Las fases fueron:

1. Audit de la hoja de valoración antes de las intervenci ones (anexo I) . Estudio descriptivo transversal con una muestra de 578 hojas de valoración co-

\section{Hoja de recogida de datos}

\begin{tabular}{|c|c|c|}
\hline NHC: & & \\
\hline 3. Mes y año: & & \\
\hline 4. Letra clara: & Sí & NO \\
\hline 5. Identificación del paciente: & Sí & NO \\
\hline 6. Fecha y turno en el momento ingreso: & Sí & NO \\
\hline 7. Firma legible: & Sí & NO \\
\hline 8. Procedencia: & Sí & NO \\
\hline 9. Motivo de ingreso y/o diagnóstico médico: & Sí & NO \\
\hline 0. Alergias: & Sí & NO \\
\hline 1. Antecedentes: & Sí & NO \\
\hline 2. Hábitos tóxicos: & Sí & NO \\
\hline 3. Constantes vitales: & & \\
\hline 13.1 PA: & Sí & NO \\
\hline 13.2 FC: & Sí & NO \\
\hline 13.3 Temperatura: & Sí & NO \\
\hline - Necesidades básicas: & & \\
\hline 14.1 Respiración: & Sí & NO \\
\hline 14.2 Alimentación: & Sí & NO \\
\hline 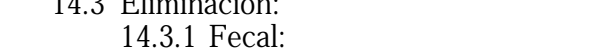 & sí & NO \\
\hline 14.3.2 Urinaria: & Sí & NO \\
\hline 14.4 Aseo e higiene: & sí & NO \\
\hline 14.5 Movilidad: & Sí & NO \\
\hline 15. Seguridad: & sí & NO \\
\hline 16. Integridad de la piel: & Sí & NO \\
\hline 17. Sentidos & & \\
\hline 17.1 Vista: & Sí & NO \\
\hline 17.2 Oído: & Sí & NO \\
\hline 18. Prótesis: & Sí & NO \\
\hline 19. Aspectos psicológicos y sociales: & Sí & NO \\
\hline
\end{tabular}




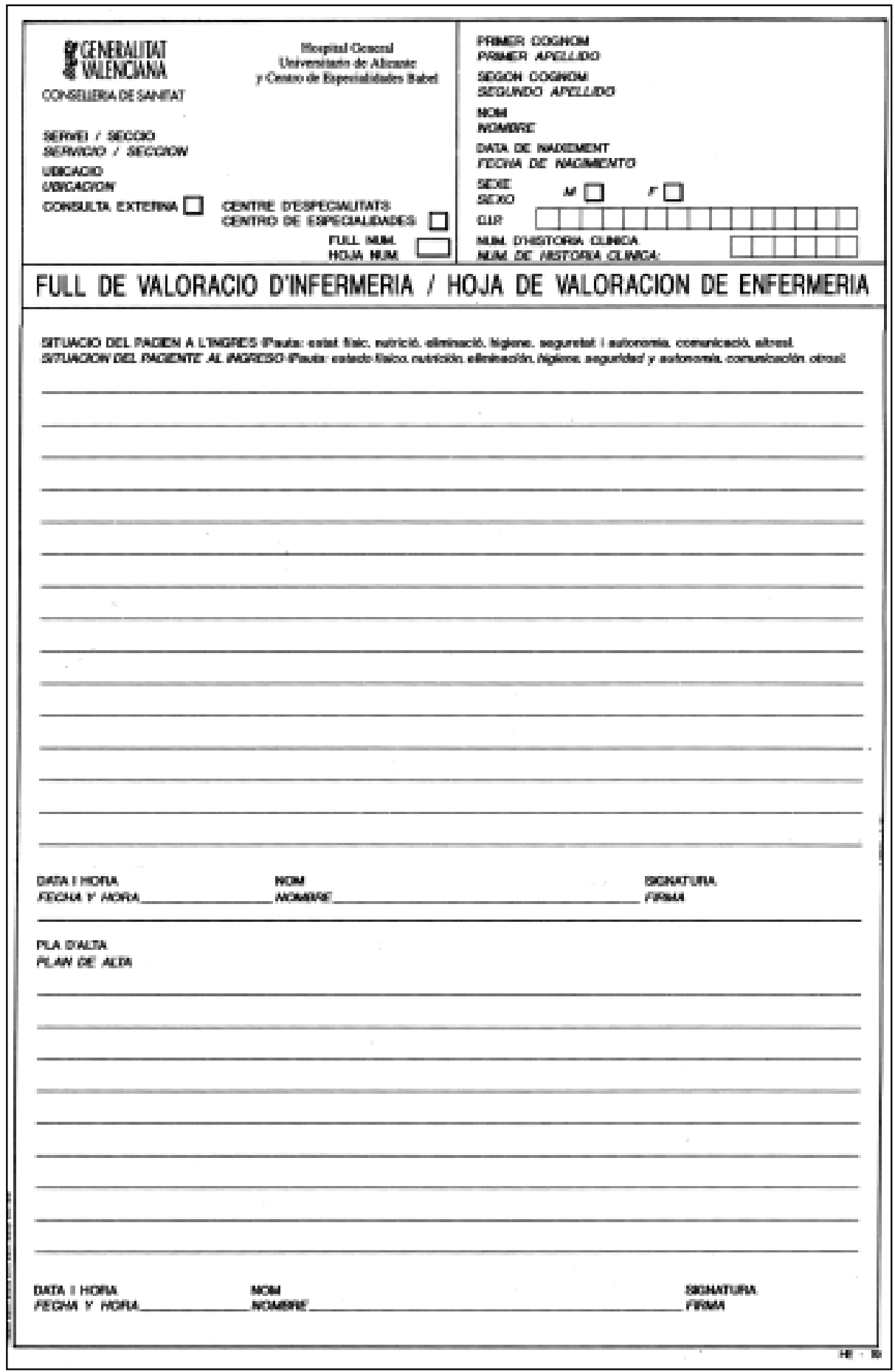

ANEXO I.

rrespondientes a todos los pacientes ingresados en el HGUA que se realizó el día 3 de febrero de 2000. Para la recogida de datos se diseñó una hoja ad hoc. Se elaboró una base de datos utilizando el programa D-base 3 y se procedió a un análisis por frecuencias relativas.

2. Intervenciones. Trabajo conjunto con los profesionales enfermeros para el establecimiento de las causas del bajo nivel de cumplimentación y las posi- bles intervenciones ( grupo nominal y cuatro grupos de discusión) $)^{9,10:}$

a) Grupo nominal: compuesto por 8 profesionales. De ellos, 5 eran mujeres y 3 hombres; a la hora de la selección se tuvieron en cuenta características como la edad y la formación académica de los participantes. Se escogieron de diversos intervalos de edad. Así se seleccionaron ATS y DUE debido a que esta situación académica está presente en nuestro colectivo. Se realizó una sesión con una duración de 2 horas. La pregunta a debatir fue: "¿Cuál es, en su opinión, la principal causa que provoca el bajo nivel de cumplimentación de la hoja de valoración del paciente al ingreso?". Dados los resultados obtenidos en esta fase, se programaron 4 grupos de discusión para analizar las posibles soluciones para cada una de las causas detectadas.

b) Los grupos de discusión estaban formados por 8 enfermeros asistenciales, a los que se les propuso el tema a debate según los resultados de la fase anterior. Los temas fueron:

- Baja autoestima profesional.

- Falta de conocimientos para plasmar por escrito el trabajo.

- Inadecuada infraestructura en las unidades y falta de tiempo.

- Inadecuado formato de la hoja de valoración vigente.

- Diseño de la nueva hoja de valoración (anexo II) y su guía de cumplimentación. En la nueva hoja de valoración se introducen nuevas variables con el objetivo de ampliar aspectos específicos de determinadas necesidades: preferencias alimentarias y dieta, patrón de eliminación fecal, patrón de descanso, estado de higiene, cuidador principal y teléfono de contacto. También se añadieron a la valoración las escalas de Estatus Funcional de la OMS y la escala de Braden 


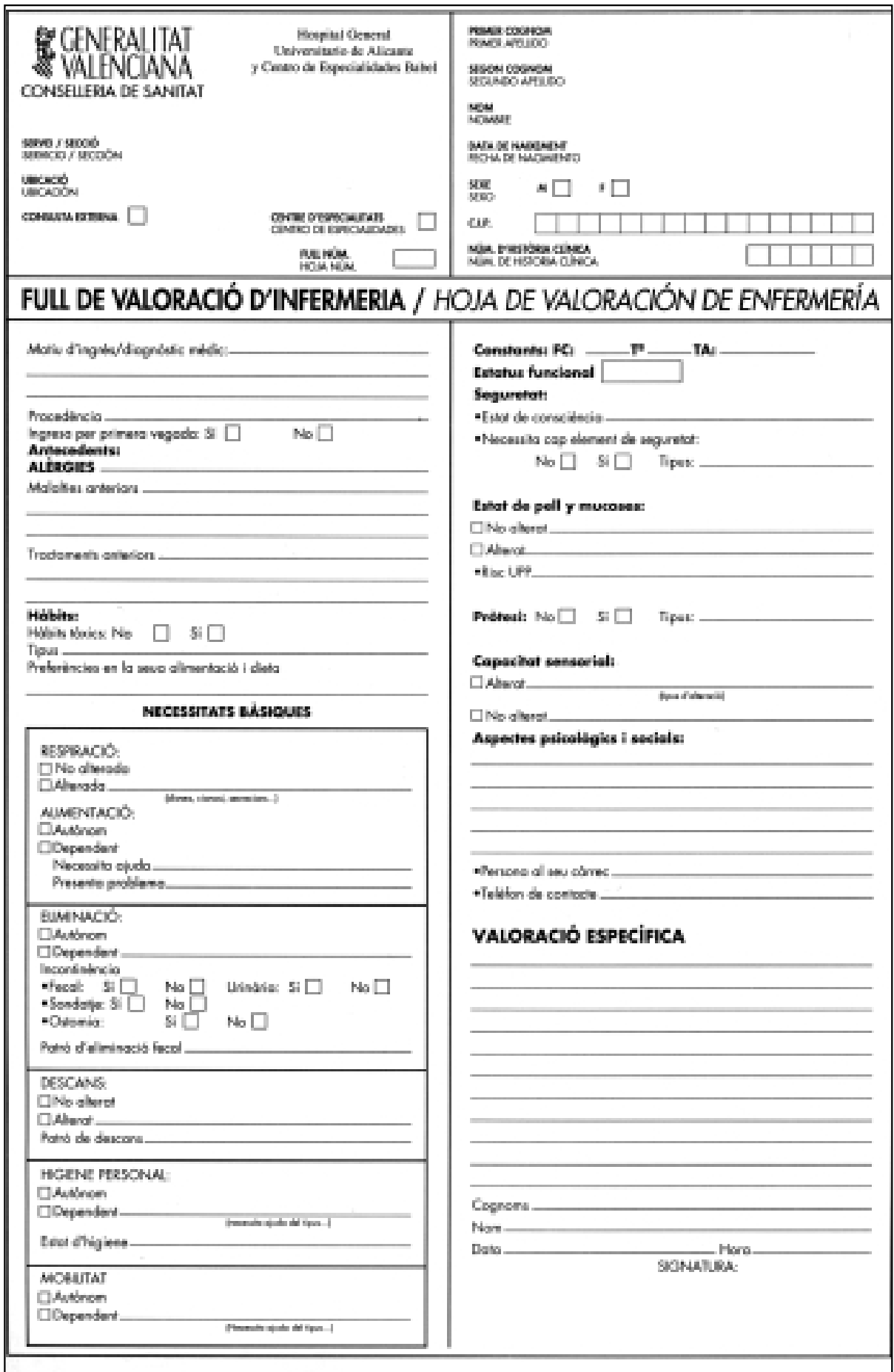

ANEXO II.

para la valoración de riesgo de úlcera por presión, por ser 2 escalas de gran importancia para el cuidado del paciente hospitalizado.

- Implantación de la hoja y su guía en la unidad piloto (tras seminarios formativos). Implantación de la hoja y su guía modificada en todas las unidades de hospitalización tras los seminarios formativos para todos los profesionales de enfermería en julio de 2001.
3. Audit después de las intervenci ones. Por último, se realizó un estudio descriptivo transversal, con una muestra aleatoria de 104 hojas de valoración de pacientes ingresados el día 20 de septiembre de 2001. Para la recogida de datos se utilizó la propia hoja de valoración ${ }^{11-17}$. Se elaboró la base de datos en Access y se procedió al análisis comparativo de frecuencias en los estudios antes-después.

\section{Resultados}

Los resultados de este estudio se pueden dividir en 3 fases. En principio, nos centraremos en la descripción de los datos más relevantes obtenidos en el estudio descriptivo transversal realizado el día 3 de febrero de 2000 ( tabla 1).

- Los porcentajes de cumplimentación correspondientes a las necesidades básicas son los siguientes:

Respiración: 23,7\%.

Alimentación: 37,5\%.

Eliminación: $36,7 \%$.

Aseo e higiene: $11,1 \%$.

Movilidad: 17,1\%.

Los porcentajes de los ítems de los datos administrativos del paciente esta-ban cumplimentados en un $92,4 \%$ de las hojas de valoración. El motivo de ingreso y el diagnóstico médico aparecían en un $92,7 \%$ de los casos. Las alergias, en un $43,6 \%$. En cuanto a las constantes vitales los porcentajeseran los siguientes:
Frecuencia cardíaca : $3,6 \%$.

Temperatura: $17,6 \%$.

Presión arterial: 27, 9\%.

La valoración del estado de la piel y las mucosas del paciente se reflejaba en $11,4 \%$, y los aspectos psicológicos y sociales, en el $10,9 \%$. 
TABLA 1. Porcentajes de ítems correctamente valorados antes y después de las distintas intervenciones

\begin{tabular}{|l|c|l|}
\hline Variables & $\begin{array}{c}\text { Valorados } \\
\text { antes \% }\end{array}$ & $\begin{array}{l}\text { Valorados } \\
\text { después \% }\end{array}$ \\
\hline Identificación del paciente & 9,4 & 96,5 \\
Motivo de ingreso & & \\
o diagnóstico médico & 92,7 & 98,8 \\
Procedencia & 56,2 & 78 \\
Ingresa por primera vez & & 73,3 \\
Alergias & 43,6 & 89,3 \\
Enfermedades previas & 49,5 & 89,53 \\
Tratamientos previos & & 69,7 \\
Hábitos tóxicos & 6,1 & 88,37 \\
Preferencias alimentarias & & \\
y dieta & & 51,16 \\
Necesidad. básica: respiración & 23,7 & 95,3 \\
Necesidad. básica: alimentación & 37,5 & 93,2 \\
Necesidad. básica: eliminación & 36,7 & 96,5 \\
Patrón de eliminación fecal & & 34,9 \\
Descanso & & 86 \\
Patrón de descanso & & 33,7 \\
Nec. básica: aseo e higiene & 11,1 & 90,74 \\
Estado de higiene & & 39,5 \\
Nec. básica: movilidad & 17,1 & 89,53 \\
Frecuencia cardíaca & 3,6 & 24,4 \\
Temperatura & 17,6 & 40,7 \\
Presión arterial & 27,9 & 74,4 \\
Estatus funcional & & 64 \\
Estado de conciencia & 32,4 & 76,74 \\
Elementos de seguridad & & 73,25 \\
Estado piel y mucosas & 11,4 & 73,25 \\
Riesgo de úlceras por & & \\
presión & & 74,4 \\
Prótesis & 3,3 & 84,88 \\
Capacidad sensorial & 3,2 & 84,88 \\
Aspectos psicológicos & & \\
y sociales & 10,9 & 37,2 \\
Cuidador principal & & 43 \\
Teléfono de contacto & & 43 \\
Valoración específica & & 72 \\
Identificación enfermera/o & 44,1 & 83,7 \\
Fecha y turno & 61,8 & 87,2 \\
Firma legible & & 87,2 \\
\hline
\end{tabular}

La identificación de la enfermera aparecía en el $44,1 \%$ de las hojas de valoración revisadas.

- Los resultados extraídos del grupo nominal fueron:

1. Minusvaloración de las tareas propias, tanto por parte de los agentes externos como de los propios compañeros y también uno mismo. Siempre se antepone la presión de las tareas delegadas que dificulta la realización de las tareas propias de enfermería.

2. Dificultad en plasmar por escrito nuestro trabajo por falta de costumbre y desconocimiento del lenguaje apropiado.

3. La infraestructura de las plantas, "las tareas intelectuales".
4. Sobrecarga asistencial que deja a la enfermera sin tiempo para sentarse a escribir.

5. Formato de hoja no facilitador; sería preferible una hoja semiestructurada.

6. Ausencia de refuerzo-motivación.

- Los resultados obtenidos de los grupos de discusión fueron:

1. Grupo de baja autoestima profesional: No hay criterios unificados para la valoración, sería aconsejable la unificación de parámetros para todo el hospital, por medio del uso de una misma hoja de valoración en todas las unidades o de una guía para su cumplimentación.

Se plantea la razón de separar el registro de enfermería de la historia general del paciente, para comodidad de las enfermeras: si se une a la historia general, escribirán menos (menos accesibilidad a la historia en cualquier momento).

Hay muchas enfermeras que escriben, pero lo hacen de forma individual, se han enseñado a sí mismas; hay enfermeras que no lo hacen porque no les gusta escribir, o les cuesta, no lo viven como algo necesario o no se les da bien. Algunas sólo escriben el relevo y lo más llamativo, aquello que luego puede dar problemas, para cubrirse las espaldas. "No sentimos lo realmente importantes que somos... Sin nosotros no habría un seguimiento individualizado y continuo del paciente."

"La verdad es que lo que se escribe no se utiliza, no vale para nada, no lo ve nadie, siempre te tienes que fiar de lo que haces tú, a saber lo que hace el otro turno... es difícil convencer a la gente de que escriba cuando en realidad no sirve para nada. Sin embargo, es muy importante saberlo todo sobre el paciente, si no cómo sabes cómo te llega un paciente que te trasladan de otra planta..." "E ntonces, sí que es necesario escribir para que lo lea el compañero..." "E so es facilitarle la labor al compañero." "Todos escribimos, aunque sea poco, en el relevo de enfermería, para el seguimiento del paciente por el turno siguiente, para facilitar el trabajo." "La enfermera siempre se ha caracterizado por proteger al paciente, y el médico por protegerse a sí mismo... Debemos hacer que cambie el chip de la enfermería un poquito hacia nosotros mismos, pero sin perder de vista al paciente... La dirección debería dar soporte, crear foros de debate, hacer algo para que le enfermera se sienta segura, que su trabajo tiene una línea de seguridad y continuidad." "Yo creo que no debemos centrarnos en que escribir ayuda a otros profesionales, yo creo que debo pensar en que mis compañeros van a reconocer mi trabajo, mis propios compañeros y yo mismo, que me siento útil y respondo a las necesidades del paciente." 
"Cuando uno deja por escrito lo que valora, o lo que hace con el paciente, evita los errores orales y los malentendidos porque por escrito se tiene más precaución, por lo tanto somos más eficaces al disminuir los errores."

"Pero hay algo más, hay que creer en lo que estás haciendo, hay que darse cuenta del valor del reconocimiento personal y profesional." "Muchas veces no hay interés en empezar a escribir, porque la enfermera ha sido autodidacta en cuanto a los registros, por lo menos nuestra generación, las más jovencitas ya no tanto, aunque luego las devora la rutina del trabajo en planta." "Mira, otra cosa es que, o escribimos lo que hacemos, o cómo reivindicamos las cargas de trabajo, no se refleja lo que se hace, sólo el paciente sabe lo que hemos hecho, porque no está escrito para recuperar la información." "Es la base de la investigación para nuestra profesión, y la única forma de protección legal."

"Nos falta cultura de grupo, sentarnos a mirarnos las caras, y a hablar de nuestro trabajo, llámalo sesiones de enfermería, o como quieras, pero cosas como las de hoy nos hacen falta en el hospital, y mucho."

2. Conocimi entos: "Nunca han tenido demasiado interés en que sepamos escribir bien o en que usemos una terminología culta, pienso que, para el estamento médico, el que no sepamos demasiado nos hace más obedientes." "La enfermería ha sido hasta hace poco autodidacta, y eso no está bien." "Perdona, pero si se escribe algo es gracias al esfuerzo de los antiguos titulados, que no han tenido ninguna formación y que se han esforzado por hacerlo." "Lo que está claro es que existen dos clases de enfermeras: las de las teorías, que me parecen muy bien, que es la de la investigación, las que están en la universidad, y luego la que trabajan a pie de cama, que no entiende de filosofías ni de investigación, y que como mucho han oído hablar de Florence Nightingale, 0 de Henderson, por lo de las necesidades que todos más o menos manejamos, pero que no les pidas nada más... Y eso no quiere decir que sean malos profesionales, hay gente muy buena que no sabe escribir y no lo hace, y punto." "Tenemos que ponernos de acuerdo en un lenguaje claro, nuestro, que nos ayude a comunicarnos... Mira cómo en los relevos orales todos nos entendemos... pues igual, pero por escrito..." "La única forma de solucionar esto es mediante una buena oferta de formación continuada y continua en registros, pero a la que asistamos los enfermeros... y para eso hay que estar motivado."

"La gente se queda tan tranquila pensando que rellena las hojas, como por trámite, y ojo que les digas algo, aunque entre otras cosas, las viejas glorias... que son las autodidactas, se sienten en poder de la verdad absoluta... Yo no sé si es por eso o porque están quemadas, pero no hay quien las haga cambiar; al contra- rio, si te ven sentada escribiendo, te pegan cada mirada que..." "Pues la nueva hornada de DUE terminará haciendo lo mismo, porque el trabajo te come, aunque es cierto que su formación no es la nuestra, tendréis que esperar a que nos jubilemos..." "Hablando en serio, una hoja semicerrada, donde se pudiera anotar de forma sencilla, facilitar el paso intermedio, aparte de la formación, ¿no sería adecuado a nuestra situación?" "Pues sí, porque a veces, cuando utilizamos algún término, yo entiendo una cosa y mi compañera otra..." "O el uso de escalas, o la definición general de los términos que vayamos a emplear anotados en una guía..." "Las guías ya existen y no se las lee nadie desde el año catapún, y no hablemos de los protocolos en planta ... (murmullos de desacuerdo) ... bueno, al menos en algunas plantas." "Deberíamos actualizar todo lo que utilizamos por escrito, todos los indicadores, las escalas, aquí la que se está continuamente actualizando es la de las escaras, los supervisores se encargan a todas horas, y eso es porque nos interesa, pero sólo en las plantas donde se tiene más pacientes encamados, no en todo el hospital." "Que tenemos que hablar más y ponernos de acuerdo en lo que escribimos y no hay nada como hablar para entenderse."

3. Infraestructura y ti empo: "Se necesita un sitio específico donde haya tranquilidad para poder escribir, porque el control no es para escribir: suena el teléfono, te llaman de la habitación..." "Sí, pero ¿de dónde sacas tiempo para meterte en un despacho para ponerte a escribir? Yo no puedo dejar a mi compañera sola con toda la planta durante media hora para irme a escribir."

"Se debe estar más tiempo con los enfermos." "Se necesita nuestra independencia con respecto a los médicos, porque nos delegan muchas tareas." "EI descui do de los médicos acumula trabajo, y si no vas haciendo de secretaria, eres desobediente... Incluso en algunos servicios se les rellenan las peticiones... La secretaria es del servicio, y no del médico." (Se comenta cómo poder afrontar el problema con los médicos, y cómo lo han hecho los servicios donde ya lo han superado.) "Si todos nos ponemos de acuerdo en qué tenemos que hacer y qué tenemos que tolerar, y si la dirección nos apoya en el tema, se supera. Lo malo es que en algunos servicios estamos desamparados y terminas pidiendo el traslado o quemándote, y siguen con el mismo tipo de enfermería que hace treinta años". "Desde siempre enfermería asume las tareas que no son de nadie, y además es que nos lucimos, lo hacemos todo estupendamente..." "La de tiempo que ganaríamos si nos quitáramos de encima las tareas administrativas: ordenar las historias de los pacientes, pedir pegatinas, reclamar analíticas, pasar visita con el médico..." "Mira, lo de pasar visita, depende de cómo se haga en la planta. Desde luego, pasar visita para llevarles las carpetas o 
cosa semejante, no, pero si es una visita conjunta donde los dos profesionales entren a ver al paciente como equipo, donde puedas opinar, ofrecer tu punto de vista como enfermera, entonces sí." "Tenemos miedo al médico, es un tema de años... a veces la falta de respeto del médico frente a la enfermera, incluso delante del paciente... te comen, te comen."

"La independencia profesional se consigue si somos capaces de reivindicar nuestro tiempo para nuestras tareas y viceversa, trabajamos con método, nos motivamos si somos reconocidos por nosotros mismos... Intentar introducir algo por narices es un horror..." "Cada planta, según su ritmo de trabajo debería tener su tiempo preestablecido... Si nos centramos en la hoja de valoración, hacerla en el momento del ingreso, con el paciente, en la habitación del paciente, al mismo tiempo que te presentas, es una buena forma de entrar en contacto..." "Si tienes que escribir o anotar primero, y luego pasar a limpio, pierdes tiempo."

"Es necesario que todo el equipo que trabaja con el paciente comparta todos los recursos de la planta, y si hay despachos para escribir o salas para reuniones, que no ponga en la puerta que es propiedad privada de los médicos..." "Pero no deberíamos estropearlo nosotros mismos, porque si te dan el uso de un despacho y terminas utilizándolo para ir a dormir... o para tomarte el café en privado..."

4. Formato hoja: Se inicia la reunión comentando la necesidad de usar un lenguaje común, propio de enfermería, "porque no somos médicos, aunque nos tengamos que entender con ellos, pero como equipo." "Creo que la hoja de valoración actual es demasiado abierta, te deja en el aire... sería bueno cerrada". "Sí, pero no cerrada del todo, que debemos manejar vocabulario... no está bien que nos dediquemos a poner cruces sin más, vamos a calentarnos un poco la cabeza..." "Vale pues con algunos puntos que sean cerrados y otros abiertos, un formato mixto." "Una guía ayudaría bastante, pero una guía que se encontrara en el mismo sitio donde haces la valoración, que no tuvieras que buscarla a todas horas y que no fuera un 'tocho', sino algo sencillo: dos, tres folios..." "Esa guía serviría para unificar criterios y también el uso del vocabulario, con definiciones operativas de cada ítem..." "Debemos acostumbrarnos a utilizar índices y escalas, incluso dependiendo de cada unidad, pero que todos los conociésemos." "Para eso sería bueno realizar sesiones monográficas de registros, para actualizar conocimientos... y así nos comunicamos cosas de nuestro trabajo... Hace tiempo estuve en una planta donde se hacían sesiones de enfermería y era una maravilla hablar de nuestro trabajo, porque mientras te tomas el café desde luego no hablas igual del trabajo, aunque comentes cosas de los pacientes."
- Los resultados más representativos del audit realizado después de las intervenciones (tabla 1 ):

Los porcentajes de cumplimentación de las necesidades básicas:

Respiración: 95,3\%.

Alimentación: 93,2\%.

Eliminación: 96,5\%.

Aseo e higiene: $90,74 \%$.

Movilidad: $89,53 \%$.

Los datos administrativos del paciente aparecían en un $96,5 \%$ y el motivo de ingreso, en un $98,8 \%$ de las hojas de valoración auditadas. Las alergias aparecían en un $89,3 \%$.

También aumentaron los porcentajes de las constantes vitales:

Frecuencia cardíaca: $24,4 \%$.

Temperatura: $40,7 \%$.

Presión arterial: $74,4 \%$.

Asimismo, los porcentajes de las nuevas variables introducidas fueron:

Preferencias alimentarias y dieta: $51,16 \%$.

Patrón de eliminación fecal: 34,9\%.

Patrón de descanso: $33,7 \%$.

Estado de higiene: $39,5 \%$.

Estatus funcional: $64 \%$.

Riesgo de úlcera por presión: 74,4\%.

Cuidador principal: $43 \%$.

Teléfono de contacto: $43 \%$.

La identificación de la enfermera aumentó hasta un $83,7 \%$.

\section{Discusión}

A la vista de los datos obtenidos podemos observar un aumento considerable en el nivel de cumplimentación de la hoja de valoración enfermera del paciente al ingreso, con lo que se confirma nuestra hipótesis de trabajo.

Es de destacar el aumento en la cumplimentación de aquellos ítems que pertenecen al campo de actuación propio de las/os enfermeras/os, como son las necesidades básicas.

Al inicio sólo llegaban al nivel adecuado de cumplimentación (mayor que $90 \%$ ) los ítems del paciente y motivo de ingreso y diagnóstico médico. Creemos que esta circunstancia se debe al valor que la enfermera daba a estos ítems para su trabajo, pues sólo éstos le servían para su tarea asistencial y nunca podían faltar. Sin embargo, en la nueva hoja de valoración, además de estos 2 ítems, que también 
experimentan una mejora, se ven adecuadamente valorados los correspondientes a las necesidades básicas, otras necesidades (termorregulación, estado de los tegumentos, aspectos psicológicos y sociales...), las alergias y enfermedades previas.

La elección de la escala de Braden, en vez de la de Norton u otra de las existentes, se debe a que ya se encontraba dentro del protocolo de prevención de úlceras por presión del hospital, creada por una comisión especial, aunque no se encuentra implantada en la totalidad de las unidades y su uso no está estandarizado.

E ntre las nuevas variables contempladas en esta hoja, es decir, las que introducen aspectos específicos en determinadas necesidades, cabe destacar la utilización de las escalas, que con anterioridad era inexistente. $Y$ así, vemos que el estatus funcional es utilizado en el $64 \%$ de las valoraciones y la escala de Braden, en el $74 \%$. Estas escalas han sido muy bien acogidas por los profesionales, como corroboran estos datos, y no se observa oposición a este cambio.

El aumento en el nivel de cumplimentación ha sido posible gracias al trabajo conjunto con los profesionales de enfermería de este hospital, ya que a diferencia de otros trabajos similares recogidos en la bibliografía ${ }^{18,19}$ en éste para todas las intervenciones se parte de su opinión y su participación en la toma de decisiones; el resultado obtenido es superior en este estudio que en los ya referenciados. Los profesionales participaron tanto en la priorización de las causas de la baja cumplimentación como en la selección de las estrategias de mejora. Al reflexionar sobre esta participación en la toma de decisiones, los propios participantes la calificaban de estimulante y motivadora hacia la realización de tareas de mejora.

Tras la realización de la técnica de grupo nominal para la detección de las causas de la baja cumplimentación, la primera conclusión que se obtuvo fue la necesidad de continuidad en el trabajo conjunto con los profesionales de enfermería, que iban a ser los protagonistas y los actores del posible cambio. Si reflexionamos sobre lo ocurrido durante el grupo nominal, y los comentarios realizados por los participantes, vemos que el sentimiento de ser escuchados, de sentirse con voz, y a su vez escuchar al compañero era un refuerzo clave para la consecución del objetivo del proyecto. De esta forma, el investigador, al intervenir, ya estaba favoreciendo un cambio, útil para su finalidad 20 .

La necesidad, como hemos indicado, de continuidad en el hecho de servir como simple catalizador al servicio de la necesidad de cambio del profesional de enfermería, al mismo tiempo que la posibilidad de ampliar el conocimiento de campo sobre la cuestión, nos animó al cambio de estrategia, programando y planificando los grupos de discusión posteriores a la técnica del grupo nominal, que dieron entrada a 32 profesionales más.

La hoja de valoración de enfermería fue la puerta de acceso a temas clave de la praxis enfermera; cada una de las causas obtenidas es aplicable a la generalidad, al origen de nuestra perspectiva actual, 0 al menos explicativa de nuestra situación de evolución profesional y, sobre todo, de nuestra evolución científica. La división entre enfermería asistencial y enfermería teórica es un hecho tal que en ningún momento de las discusiones de trabajo se comentó la existencia, o posible utilización o adecuación, de modelos teóricos aplicables a la praxis enfermera que solucionaran el problema de la no escritura, ni mucho menos que sintiera la necesidad de adoptar un modelo de enfermería como posible solución a su situación profesional, en cuanto a la relación con el paciente o con el resto de su entorno ${ }^{21,22}$. Los profesionales reconocen su falta de formación en cuanto a la cultura propia de la profesión, con todo lo que ello conlleva en cuanto a la falta de identidad asumida. Nunca se podrán adoptar medidas de mejora en aspectos que se desconocen. Por todo ello, la adopción de un determinado modelo de registro tendrá que adaptarse al momento profesional y a las características propias de los profesionales que lo integran. Un modelo de registro abierto da pie a la no escritura en este momento; hay que promover un cambio cultural, de hábitos, de conocimientos, de vivencia del trabajo individual como parte del trabajo profesional de un colectivo que se reconoce en cada uno de sus miembros.

\section{Bibliografía}

1. Gutiérrez de Terán Moreno G. Aspectos legales de los registros de enfermería. Enfermería Clínica 3(1):21-4.

2. Marc Mandell J D. Si no se ha registrado es que no se ha valorado. Nursing Mar, 1995.

3. Aparicio Casals, Martorell Poveda A. Registros de enfermería en atención primaria: pieza clave para la investigación. Enfermería Científica, 1993;133:11-4.

4. Amezcua M. La calidad en la documentación clínica de enfermería: del papeleo a los registros para el cuidado. Index de Enfermería 1995;11:15-20.

5. Diesser M, Feingold L, Rosenkranz J L, Coltin KL. Clinical quality measurement. Comparing chart review and automated methodologies. Medical Care, 1997;35:539-52.

6. Topp M, Langhoff-Ross J, Uldall P. Validation of a celebral Palsy Register. Clinic E pidemiology 1997;9:1017-23.

7. Tara Massie J. Implementation of change: a wound assessment chart. Professional Nursing 1998;14:2.

8. Pike, Kenneth. Language in Relation to a Unified Theory of the Structue of Human Behavior. Santa Ana, California: Institute of Linguistics, 1954.

9. Ivonne Lincoln S. Conexiones afines entre los métodos cualitativos y la investigación en salud. Investigación y adecuación en enfermería. Medellín XV (2), 1997; p. 57-69.

10. J osé I Ruiz Olabuénaga. Metodología de una investigación cualitativa. Bilbao: Universidad de Deusto, 1999.

11. Herrero García T, Cabrero Cabrero AI, Burgos Martín MR, García Iglesias M, Fernández Herrainz Al. Control de calidad en los registros de enfermería. Enfermería Intensiva 1998;9(1):10-5.

12. Córcoles ] iménez MP, García Piñero MP, Ruiz Gómez P, Pita Toledo $T$, Marín Cortés L. El dossier de enfermería en el complejo hospitalario de Albacete: composición y calidad. Revista Rol 1997;1:19-24. 
13. Miró Bonet M, Amorós Cerdá SM, De J uan Sánchez SM, Fortea Cabo J, Fran Morro E, Moragues Mas J. Valoración al ingreso del paciente crítico. Un indicador de calidad asistencial. Enfermería Intensiva 2000;11(2):51-8

14. Alonso González M, García de San J osé S, Díaz González L, García Caballero ]. Valoración de la calidad de las historias clínicas. Gestión Hospitalaria 1996:4:44-50.

15. Polo Tolosana M, García Pardos A, Mairal Salinas EL, Oliván Esco MP, Les Macho A, Vimales Palomar C, et al. Registros: Revisión de la documentación clínica de enfermería de un hospital general en el año 1994. Enfermería Clínica;7(3):72-9.

16. Romera Rufián PF. GARE. Guía de auditoría de los registros enfermeros. Index de Enfermería 1993;6:36-40.

17. Corrales Nevado D, Rubio Villegas C. Protocolización y registro en atención primaria: a propósito de una hoja de monitorización. Aten Primaria 1994;13(4).
18 Blanco Rodríquez J M, Rodríguez J iménez A, De Luis Lozano A, Rodríguez Pita D, Gómez Auñón J, Fernández Martínez A, et al. Control de la calidad del registro de valoración de enfermería al ingreso. Enfermería Científica 1997;184-5.

19. Besora Torradeflot I. Implantación de una hoja de valoración de enfermería en Atención Primaria. Enfermería Clínica 4(3):44-6.

20. Siles J . E pistemología y enfermería: por una fundamentación científica y profesional de la disciplina. Enfermería Clínica 1997;7(4).

21. Tomás Vidal AM, Sánchez Flores MI, Torres Puerto M. Desarrollo de un formato de valoración por patrones funcionales de salud. Enfermería Científica 1992;128:18-21.

22. González Carrión P, Hernández Padilla M, Rivas Campos A, Toral López I, Sánchez Martínez MP, Quero Rufián A. Validación de los diagnósticos de enfermería de la NANDA: una experiencia en cuatro áreas asistenciales. Enfermería Clínica 4(3):124-7. 